\title{
EL PSICOANÁLISIS ¿QUÉ TIPO DE CIENCIA ES?
}

PSYCHOANALYSIS, WHAT KIND OF A SCIENCE IS IT?

\section{RESUMEN}

El modelo de ciencia que la psicología académica ha adoptado se atiene casi exclusivamente a criterios empírico-experimentales. Desde este bastión se ha tratado de derruir el saber psicoanalítico, arrojándolo a la cárcel de las pseudociencias o de los mitos. En un momento determinado se intentó acercar el psicoanálisis a tal modelo positivista, lo que trajo consigo la pérdida de lo más esencial del mismo. Sin embargo, desde la década de los sesenta, algunos venimos insistiendo en ubicarlo en el marco de las ciencias histórico-hermenéuticas, donde la teoría y la práctica psicoanalíticas recuperan su total identidad. En este trabajo, de forma muy sintética, se hace un recorrido de todo lo anterior, argumentando en favor de la tesis que sitúa al psicoanálisis dentro de los saberes histórico-hermenéuticos valiéndose tanto de estrategias inductivas como deductivas.

Palabras claves: Psicoanálisis, Ciencia, Pseudociencia, Epistemología, Histórica, Hermenéutica, Deductivo, Inductivo.

\section{ABSTRACT}

The model of scientific knowledge that the academic Psychology had adopted follows, mainly, empirical and experimental criteria. From this standpoint, thus, there have been numerous attempts to devalue the psychoanalytic body of knowledge by being depicted as pseudoscientific or just a myth. Consequently, the psychoanalytic community made some efforts to drive Psychoanalysis closer to Positivism, which had as a result the loss of its essence. However, since the 60 's, some psychoanalytic authors have insisted on including the Psychoanalysis as a part of the Historical-Hermeneutic knowledge, in order for its theory and practice to recover all their identity. In our paper, we have briefly reviewed the above ideas, supporting the thesis that, by means of deductive and inductive strategies, Psychoanalysis should be placed into the Historical and Hermeutical boy of knowledge.

Keywords: Psychoanalysis, Science, Pseudoscience, Epistemology, Historical, Hemeneutical, Deductive, Inductive. 


\section{INTRODUCCIÓN}

El modelo científico actual, particularmente dentro de la Academia, es sin duda de carácter empírico-experimental, lo que implica una serie de supuestos epistemológicos, tales como los de atenerse al estudio de hechos de carácter objetivo y propiciar explicaciones de tipo causalista, o, al menos, funcionalista, basadas en presuntas leyes reguladoras de carácter general. El psicoanálisis, a pesar de los deseos de Freud de ubicarlo dentro de las ciencias naturales, no puede ser encuadrado en este marco y de aquí que haya sido sometido reiteradamente a críticas muy duras, tratando de condenarlo al infierno de las pseudociencias o de los mitos.

En tal situación, algunos psicoanalistas adoptaron variadas salidas, desde encerrarse en una torre de marfil, haciendo oídos sordos a los ataques, hasta proponer reformulaciones del cuerpo teórico psicoanalítico en una línea acorde con la perspectiva de las llamadas ciencias empíricas (opción que también intentaron autores ajenos al psicoanálisis), así como otras alternativas más interesantes, como la de fundamentarlo como un saber ajeno a tal modelo, como lo histórico-hermenéutico, pero no por ello ajeno a lo científico $(1,2,3)$.

En cuanto a las reformulaciones empiristas, la gran falla de sus defensores fue el dejar al psicoanálisis sin su alma, sin su esencia, pues tales propuestas derruían totalmente el soporte metapsicológico, su núcleo fundamental. A partir de la década de los sesenta del pasado siglo, sin embargo, se ha venido intentando una nueva forma de justificar el psicoanálisis, encuadrándolo dentro de las denominadas ciencias histórico- hermenéuticas, lo que ha permitido recuperar sus principales señas de identidad. Pero para que ello sea posible es preciso abandonar una serie de apriorismos, como el de concebir la realidad, campo de estudio de toda ciencia, como un conjunto exclusivamente formado por hechos objetivos u objetivables, incluyendo en tal realidad lo subjetivo, de imposible objetivación a través de los métodos científicos habituales. Así mismo, es imprescindible ir más allá de las explicaciones causalistas y funcionalistas, así como de las fenomenológicas, que sólo dan cuenta de las vivencias conscientes, proponiendo en su lugar explicaciones de carácter dinámico-exegéticas o interpretativas, que conceden el máximo papel al hallazgo del sentido o significado de la realidad psíquica construida en el pasado e incluso en el mismo instante de mostrarse ante el analista, a partir de los determinantes dados por la participación de los procesos inconscientes.

Antes de emprender la tarea de justificar que la teoría y la práctica psicoanalíticas adquieren un sentido adecuado dentro de tal perspectiva, hemos de recordar los fallidos intentos empíricos, ocupándonos someramente de las ideas de Bridgman, Madison, Sullivan, Rapaport, Peterfreund, Gear y Liendo, G. S. Klein y Schafer, que fueron los principales representantes de tal modo de entender el psicoanálisis. 


\section{REFORMULACIONES EMPÍRICAS DEL PSICOANÁLISIS}

Debe atribuirse a Bridgman $(4,5)$ el primer intento de convertir al psicoanálisis en una teoría empírica, para lo que se valió de la estrategia de la utilización del llamado lenguaje operacional, tan en boga en su tiempo, particularmente dentro del sistema conductista, y ello a pesar de que los filósofos ya habían abandonado entonces tal forma de trabajar en el campo científico. Bridgman trató, con ello, de dotar al psicoanálisis de unos conceptos objetivables y cuantificables, gracias a lo que poder incluirlo dentro del modelo científico imperante en su época. Su análisis operacional, sin embargo, resultó un híbrido psicoanálisis-conductismo que no permitió la puesta en marcha de programas de investigación productivos para nuestra disciplina.

Algunos años después, Madison (6) llevó a cabo una propuesta algo similar, tratando de establecer una serie de definiciones operacionales y determinadas reglas de correspondencia entre conceptos como la represión y la defensa, sin duda nucleares en el psicoanálisis, y ciertas conductas manifiestas, tratando de correlacionar el lenguaje teórico psicoanalítico con un lenguaje observacional. Tampoco este acercamiento al campo empírico tuvo éxito, no sólo a causa de la naturaleza de su compromiso epistemológico, extraño al psicoanálisis, sino también por sus marcadas limitaciones.

Ya desde dentro del sistema psicoanalítico no pocos saludaron con júbilo las aportaciones de Sullivan $(7,8)$, entendiendo que favorecían el definitivo progreso del estatuto científico del psicoanálisis, al asentarlo en el tan anhelado camino empírico, a pesar de que aquél siempre sostuviera que sólo intentaba hacer unas matizaciones para la mejor utilización de la teoría freudiana (9). En efecto, a nuestro modo de ver, lo que Sullivan llevó a cabo fue la inclusión de factores socio-culturales y una interesante renovación del lenguaje psicoanalítico para dar una explicación de la determinación de la estructura del carácter individual, creando según Chrzanowski (10), crear fundamentos epistemológicos más sólidos para las observaciones clínicas que los que se lograban con la metapsicología tradicional, así como atender a un campo transaccional más vasto, al incluir en su esquema, de cierta orientación operacional, factores culturales y sociales provenientes del medio. Por ello, puede decirse que Sullivan fue uno de los iniciadores del psicoanálisis culturalista, movimiento desde el que Horney, Fromm, Erikson y otros enriquecieron el freudismo, al considerar ingredientes que daban más juego que los incluidos en el enfoque biologista entonces existente. Pero nada de ello supuso, desde luego, entrar en los parámetros propios de las ciencias empírico-experimentales tan poco queridos por el propio Freud.

En cuanto al intento de sistematización de David Rapaport $(11,12)$ fue mucho más comprometido con la perspectiva cientifista de la época, ya que trató de encajar los conceptos psicoanalíticos básicos en un marco empirista a través de una serie de tesis, axiomas y teoremas, llegando incluso a delimitar un conjunto de variables que calificó como independientes y dependientes, según el más rancio modelo experimentalista. Rapaport reconoció pronto la inoportunidad de su proyecto, que tuvo 
como principal defecto el alejarse mucho de los hechos clínicos (9). Ahora bien, como aspecto positivo ha de subrayarse que la propuesta de Rapaport mostró ciertas posibilidades de formalización y sistematización de la teoría psicoanalítica, abriendo las puertas a la sedimentación de una psicología general de tipo dinámica.

En una línea más revolucionaria que la anterior, hemos de situar los aportes del psicoanalista neoyorquino Peterfreund, que planteó la necesidad de sustituir el modelo metapsicológico por un modelo sistémico y de procesamiento de la información congruente con la neurofisiología y otros conocimientos positivistas de su época (13, 14). Desde este enfoque se propuso el abandono de conceptos tan esenciales para el psicoanálisis como los relativos al yo, a la energía psíquica o libidinal, a los procesos primario y secundario, etc., sustituyéndolos por otros más acordes con su perspectiva, que sin duda resultaban atractivos, pero muy distantes de la visión freudiana.

Gear y Liendo $(15,16)$, por su parte, trataron de articular en el ámbito metodológico el psicoanálisis con la informática, para, según sus propias palabras, maximizar el rigor y la capacidad operativa del mismo, aplicándolo satisfactoriamente al procesamiento de los datos clínicos, con fines diagnósticos, pronósticos, profilácticos y terapéuticos. Ello exigía, naturalmente, de una serie de redefiniciones de los conceptos psicoanalíticos, con el fin de poderlos traducir al lenguaje informático y posteriormente al tratamiento matemático. Todo esto no llegó a cuajar, habiendo caído hoy prácticamente en el olvido, no sólo porque tal propuesta se alejaba absolutamente de lo que es el genuino psicoanálisis, sino también porque la simplificación que exige la informatización se torna imposible con los hechos clínicos, dada la absoluta incompatibilidad de los complejos conceptos y observaciones psicoanalíticos con los datos concretos de la informática.

Finalmente, tanto Gerard S. Klein (17) como Schafer (18) trataron de salir del atolladero que impusieron los críticos dándole la espalda a todos los elementos teóricos del psicoanálisis, proponiendo una práctica sin psicodinámica, un lenguaje en acción, que transformó el psicoanálisis en una actividad sin ninguna fundamentación conceptual, o, dicho de otra forma, en una especie de tarea artesanal.

La crítica que a todas las anteriores reformulaciones empiristas podemos hacer es que sus autores trabajaron con presupuestos más o menos desgajados del contexto clínico psicoanalítico, suponiendo además en muchos casos el abandono del más específico soporte teórico freudiano. Por otra parte, no hay que olvidar que las disciplinas psicológicas empírico-experimentales, como afirma Ricoeur (19), se ocupan de hechos que derivan de la observación directa de la conducta manifiesta, mientras que la esencia del psicoanálisis está en desenvolverse en un campo dado por la búsqueda de relaciones de sentido entre los objetos sustituidos y los objetos originarios (y perdidos) de la pulsión. El psicoanalista, ciertamente, ha de ocuparse del estudio de los derivados del inconsciente, que si bien suponen o pueden suponer un núcleo de realidad objetiva, no es en la consideración de tal núcleo donde alcanza 
sus últimos objetivos, sino en su traducción, en su exégesis, estableciendo cuál es la semántica de los deseos pulsionales en juego en el seno de las relaciones objetales (transferenciales) y siempre a la luz de los determinantes inconscientes. Por consiguiente, los aspectos manifiestos o públicos de la conducta no son sino elementos indirectos o secundarios, aunque sirvan al psicoanalista como soporte para alcanzar los objetivos primarios que guían su tarea.

En todo caso, el acercamiento del psicoanálisis a las disciplinas empíricas se establece, a nuestro modo de ver, por la consideración de ciertos acontecimientos que rodearon al desarrollo del individuo, sobre todo los que implicaron frustraciones o excesivas gratificaciones de deseos en los primeros años de la vida, circunstancias que sin duda participan en la constitución de los conflictos intrapsíquicos inconscientes. Pero lo que interesa al psicoanalista va más allá de acceder a tales reconstrucciones históricas de hechos objetivos, dado que es la realidad subjetiva, tanto la creada en el pasado personal como en el presente analítico, lo que más llama su atención. En tal sentido apunta nuestra tesis del carácter histórico del psicoanálisis, que no sólo incluye la reconstrucción de los hechos sucedidos, sino también de los fantaseados tanto en las relaciones objetales de la infancia como en las relaciones transferenciales del presente.

El psicoanálisis, además, supera la mera reconstrucción histórica, dado que busca, sobre todo, una hermenéutica de lo que el sujeto expresa en sus palabras y en su conducta. El analista está particularmente comprometido con la exégesis de la realidad subjetiva implícita en las reconstrucciones que efectúa, teniendo particularmente en cuenta lo que sucede en el campo de los fenómenos transferenciales, tratando de encontrar el último significado, el determinado por la acción del inconsciente, del acto o del fenómeno psíquico en cuestión. Por ello, podemos afirmar que, aun siendo el psicoanálisis en parte una ciencia histórica, pues la interesa reconstruir hechos del pasado, su peculiaridad está en lo hermenéutico, superando así la mera reconstrucción biográfica y las explicaciones históricas, para comprometerse en explicaciones dinámicas y profundas, en las interpretaciones, situando en primer plano los significados determinados por la influencia del inconsciente, que es a lo que podemos llamar explicaciones interpretativas o dinámico-exegéticas.

Las ideas anteriores fueron de alguna forma expresadas por los filósofos anglosajones Toulmin, Flew y Peters, captando que la explicación psicoanalítica no corresponde ni a una razón alegada, ni a una razón relatada, ni a una estricta explicación causal o funcional, aunque en el ámbito teórico tome el aire de ésta, por apuntar a causas a la hora de esclarecer el último por qué del comportamiento humano. Pero, en todo caso, los intereses psicoanalíticos apuntan a lo semántico, que se sostiene en determinantes ajenos al causalismo fisiológico o de cualquier otro tipo, estando igualmente lejos del dado por presuntas relaciones funcionales entre variables. La semántica que trata de esclarecer el psicoanálisis se refiere a la determinada por el funcionamiento dinámico del aparato psíquico, con la primacía del papel de lo incons- 
ciente, concretamente la semántica del deseo. Justamente, como ha dicho Ricoeur (19), el gran mérito de Freud fue el haber logrado llevar a un lenguaje psicológico aquellos fenómenos que en último término tienen su raíz en lo neurofisiológico (o en lo energético), es decir, en lo estrictamente causal: en efecto, la metapsicología torna psicológico lo que es energía, permitiendo conectar la explicación causalista con la explicación interpretativa.

El psicoanálisis, por todo ello, obtiene una adecuada justificación epistemológica en el terreno histórico-hermenéutico y de ninguna forma en el campo empírico-experimental, sin que por ello se trate de un saber pseudocientífico, sino científico. Ello exige ampliar la concepción de ciencia, incluyendo explicaciones que estén al margen de lo causal y funcional. Tratemos de abundar en este aserto mostrando algunas de las ideas que ya hemos expresado en otros momentos y lugares, con el fin de dar cuerpo y consistencia a esa tesis central $(1,2,3,20,21,22,23)$.

\section{EL PSICOANÁLISIS COMO CIENCIA HISTÓRICO-HERMENÉUTICA}

\section{A) el carácter histórico del psicoanálisis}

La teoría y la práctica psicoanalíticas están básicamente interesadas en la consideración del conflicto intrapsíquico inconsciente, que se constituye por los avatares de los deseos pulsionales procedentes del inconsciente en la interacción con el objeto, sobre todo durante los primeros años de la infancia, lo que sirve como fundamento, si no fueron adecuadamente resueltos, para ocasionar los diversos desajustes que el sujeto tendrá a lo largo de su vida.

Tales hechos se elaboran de una u otra manera por el aparato psíquico, dejando en la esfera de lo inconsciente las temáticas pulsionales rechazadas, más o menos conectadas con experiencias de naturaleza psicotraumática ocurridas en las primeras relaciones objetales. Ello conlleva la presencia de una cierta cantidad de energía libre, que es invertida en la organización de determinados productos caracteriales y conductuales, ya normales, ya patológicos, propiciando así la adaptación o la desadaptación. En el momento del encuentro clínico psicoanalítico, este conjunto de elementos psíquicos sufre una nueva y peculiar reelaboración en la mente del sujeto, con la participación o no de ingredientes objetivos procedentes de la interacción clínica, lo que lleva a su expresión en los llamados fenómenos transferenciales, el campo genuino del trabajo psicoanalítico.

El psicoanalista, por ello, no sólo está interesado por la interacción real o fantaseada que tuvo lugar entre el sujeto y sus padres en la infancia y por eventos puntuales que pudieran haber traumatizado psíquicamente al individuo, así como por el esclarecimiento de los deseos que en tales circunstancias pudieron movilizarse y entraron en conflicto con los controles normativos superyoicos (que en gran parte proceden del entorno cultural), sino sobre todo por lo que aparece en la relación dada por el aquí y ahora del encuentro psicoanalítico. 
En el encuadre clínico, con sus estrictas condiciones y reglas, y dentro de los llamados fenómenos transferenciales, el analizado produce una serie de contenidos (pensamientos, deseos, afectos, lapsus, sueños, actos motores, etc.) y relata una gran variedad de vivencias que le problematizan, cuyo último origen y sentido desconoce. Todo ello sirve al analista no sólo en tanto puede ser útil para reconstruir el pasado real o fantaseado, sino sobre todo en cuanto es susceptible de una interpretación profunda o dinámica, gracias a lo que podrá hacerse consciente y dominable por el yo el último sentido de los motivos de los conflictos que alteran al sujeto, de forma que el llamado proceso secundario podrá tomar el dominio donde era el dueño el proceso primario.

Como es fácil inferir de lo expresado, la reconstrucción histórica psicoanalítica no acoge sólo una recuperación de hechos objetivos del pasado y del presente, sino sobre todo de cómo se vivieron y viven subjetivamente, así como un conjunto de hechos fantaseados por el propio sujeto, siendo lo más importante tratar de darles una oportuna interpretación capaz de poner en primer plano los significados determinados por el inconsciente, recorriendo un camino que va desde el aquí y ahora al ayer y entonces.

Pues bien, en tanto el psicoanalista se compromete en la tarea de reconstruir el pasado personal en el sentido señalado, está poniendo en primer plano su vocación de historiador, mientras que se convierte en hermeneuta de lo profundo cuando efectúa una exégesis de tal pasado en el marco de su teoría y de los fenómenos transferenciales, donde la pulsión y sus avatares y la interacción con el objeto cobran la máxima importancia. En tanto historiador busca un determinado tipo de explicación y en tanto hermeneuta otra, tratando de comprender y hacer comprender la dinámica de la realidad psíquica vivida en función de los determinantes inconscientes, lo que conduce a un tipo de explicación de naturaleza ajena a la causal y a la funcional, yendo también más allá de la comprensivo-fenomenológica, de la que la estricta explicación histórica estimamos que es una forma. La explicación que maneja el psicoanalista es dinámico-exegética o interpretativa, la cual busca poner en la esfera de lo consciente lo que está oculto por la acción de los procesos inconscientes.

Concentrándonos en primer lugar en el psicoanálisis como ciencia histórica, podemos intentar una mayor profundización a partir de las ideas que nos ha aportado Gibson (24), cosa que puede facilitar el esclarecimiento de los puntos comunes que existen entre historiador y psicoanalista. Gibson indica que ser historiador quiere decir, sencillamente, que uno se interesa por lo que sucedió en el pasado. Y, en tal sentido, añadimos nosotros, el psicoanalista lo es, pues concentra parte de su trabajo en los eventos biográficos del analizado (especialmente en cómo los vivenció desde su realidad subjetiva), tratando de entresacar los claves más sobresalientes y significativas, para determinar así algunos de los factores que crearon los conflictos del individuo, teniendo siempre presente que es en las fallas del discurso verbal y no verbal del presunto recuerdo donde se expresa con más precisión lo inconsciente. 
En sus respectivas tareas, tanto historiador como psicoanalista tratan de encontrar el mayor número de pistas, para, con el máximo rigor ético y científico posibles, intentar dar una explicación de lo que aconteció en tiempos pretéritos, a la luz de sus correspondientes sistemas teóricos: en uno y otro caso se manejan explicaciones propiamente históricas, las cuales apuntan más a motivos, razones, creencias, actitudes e intereses que a causas de naturaleza fisicalista. Ahora bien, el psicoanalista supera este nivel al tratar de acceder a explicaciones de tipo dinámico-exegética (explicaciones interpretativas), pues no se da por contento con la mera comprensión de eventos de la realidad subjetiva, sino que quiere captar, para transmitirlo después, el significado profundo que todo ello ha tenido y tiene para el sujeto, significado que éste, por la acción de sus defensas inconscientes, desconoce, gracias a lo que evita la angustia que el percatarse del mismo traería aparejado.

Si profundizamos algo más en el examen de las peculiaridades de la investigación histórica, pueden determinarse más similitudes entre historiador y psicoanalista: así, en la investigación histórica destacan dos cuestiones centrales: ¿qué ocurrió? y ¿por qué ocurrió? La respuesta a la primera pregunta nos enfrenta a la estricta reconstrucción histórica, que en ocasiones puede ser conjeturada o hipotetizada, lo que puede denominarse retrodicción, una forma invertida de predicción cuando parte de un cuerpo de principios generales. La respuesta a la segunda de las cuestiones nos conduce a la explicación histórica, no pudiendo ni debiendo identificarse ésta con la explicación causal, por muchas semejanzas formales y lógicas que ambas posean.

Cuando tales retrodicciones y explicaciones son genuinas exigen imprescindiblemente de un soporte de principios generales y particulares bien establecidos. Llamar leyes a tales principios es una cuestión secundaria, aunque sería razonable reservar tal epígrafe sólo para las regularidades que subyacen tras los fenómenos que acontecen de forma cerrada y determinista, como ocurre en el terreno físico. Sea como fuere, lo que hay que resaltar es que si un quehacer viene derivado de un cuerpo de principios ( $\mathrm{y}$ por supuesto de leyes), ese quehacer es científico: y ello tiene lugar tanto en la historia como en el psicoanálisis.

Es bien cierto, sin embargo, que en los hechos históricos, y en general en la conducta humana individual, se da una circunstancia no buscada por sus estudiosos: la irrepetibilidad. Esto torna muy dificultosa la contrastación de las reconstrucciones y explicaciones en la historia y en la psicología. Es verdad que el psicoanalista tiene en este asunto ciertas ventajas sobre el historiador clásico, como es la posibilidad de comprobar más directamente si unas y otras son adecuadas, por ser más factible conectar conjeturas con eventos más o menos recientes e incluso actuales. Pero el psicoanalista también cuenta con limitaciones que debilitan su discurso científico, ya que, dado que trabaja con hipótesis-dichas, declarando a su analizado la reconstrucción que supone o la explicación histórica y la explicación interpretativa que cree que mejor se atienen a lo registrado, intervención que puede alterar la evolución 
espontánea de los datos que sirvan para confirmar o refutar las conjeturas en juego, dándose el caso de que el sujeto que las escucha se oponga aun siendo certeras, o las acepte incluso siendo incorrectas, por la intervención de factores afectivos y cognitivos que lo mediaticen en uno u otro sentido. Esto, sin duda, es una limitación epistemológica seria, que sólo puede obviarse parcialmente cuando la experiencia muestra, en muchos casos o reiteradamente en un mismo sujeto, la validez de las conjeturas que se han manejado.

Historiador y psicoanalista, por otro lado, se valen de una amplia variedad de vestigios para alcanzar sus objetivos científicos: sin tales vestigios, que en todo caso han de ser reconstruidos y descritos a la luz de una teoría, la tarea de uno y otro es inviable. La operatividad de esta labor se torna mayor, además, cuando se da una evidencia acumulativa, que puede referirse tanto al caso individual como al conjunto de objetos o sujetos que comparten la misma característica. Por esta vía se facilita la superación de lo idiográfico, accediéndose a un escalón más alto de la ciencia, lo nomotético.

Como se acaba de decir, el hallazgo y la descripción de los vestigios se realiza, inevitablemente, en el contexto de una determinada teoría. Y, paralelamente, tales vestigios pueden confirmar los supuestos teóricos, debilidad epistemológica propia de las estrategias inductivas, de alguna manera presentes en la historia y en el psicoanálisis. Igualmente es cierto, desde luego, que una y otra pueden trabajar al modo deductivo, en cuyo instante se sitúan en un nivel epistemológico de superior categoría, pero tampoco esta alternativa garantiza el encuentro con la verdad (23).

Ahora bien, debe tenerse muy presente que, tanto en la historia como en el psicoanálisis (y en toda psicología humana), cualquiera que sea la estrategia investigadora (inductiva o deductiva), las validaciones de conceptos, hipótesis o principios (generales y particulares) tienen un carácter abierto y probabilístico, como corresponde a los eventos en que participa el hombre, dado que sus actos se ponen en marcha y se sostienen por deseos, propósitos, intereses, actitudes y otros elementos psicológicos, y no directamente por causas físicas, que son las que ocasionan una determinación más cerrada, más predecible. Y de aquí que sólo en este campo podamos hablar de genuinas leyes.

Ahora bien, aunque lo histórico sea importante en el trabajo psicoanalítico, lo esencial está en la tarea hermenéutica, terreno en el que los errores son más factibles, por su propia naturaleza y por la metodología que manejamos al respecto.

\section{B) El carácter hermenéutico del psicoanálisis}

Tal como afirma Suárez (25), Ricoeur ha llevado a cabo un trabajo lúcido, riguroso y honesto para fundamentar epistemológicamente el psicoanálisis, contrastando el valor del discurso freudiano a la luz de la hermenéutica. Para Ricoeur (19) el avance epistemológico central del psicoanálisis freudiano es que supera lo energético por 
medio de lo hermenéutico, gracias a lo que lo físico se abre a lo psicológico. Este hecho constituye, justamente, la razón de ser del psicoanálisis, en donde la energética pasa por una hermenéutica y ésta describe una energética.

Ciertamente, la perspectiva metapsicológica de Freud, en especial el punto de vista económico, se enraíza en lo biológico, en lo neurofisiológico, en lo energético. Pero, sin duda, el psicoanálisis trata de ir más allá de lo somático, buscando el sentido o significado personal (y también general) del deseo pulsional y de los productos más o menos ligados a él, como las temáticas que se establecen en las relaciones con el objeto, entrando en ese instante en un terreno puramente psicológico. Aquí la aportación metapsicológica dada por la perspectiva dinámica cumple un papel definitivo, pues permite acceder a lo hermenéutico, lo que supera la reconstrucción y la explicación históricas.

Las interrelaciones existentes entre lo histórico y lo hermenéutico no chocan en absoluto, puesto que ambas tareas se complementan, particularmente en el psicoanálisis. Lo que sí queda fuera de los intereses psicoanalíticos son los estudios fisiológicos, aunque en ningún caso se abandone la idea de que lo psíquico se funda en último término en un sustrato biológico: de aquí la inclusión de construcciones económicas en la metapsicología. Pero, ¿cómo es posible ligar la explicación económica, de carácter causalista, a la interpretación de significados? Para Ricoeur (19) el freudismo existe justamente porque supera este dilema, yendo más allá de la dicotomización cuerpo-mente que subyace en la fenomenología: gracias al modelo metapsicológico, la interpretación psicológica puede surgir, en un momento dado, por encima de la explicación causalista o económica. En efecto, el aparato mental conceptualizado por Freud, sobre la base de sus características estructurales, económicas, dinámicas y genéticas permite superar el dualismo inserto en el hecho de defender una explicación causalista-fisicalista versus una explicación interpretativapsicológica, dado que tal aparato es descrito como un escenario donde la pulsión fisiológica se transforma en representación psíquica (fantasía, deseo), esto es, en pulsión psíquica, a partir de cuyos conflictos se construyen los rasgos de carácter y el comportamiento manifiesto, que esconden un significado o sentido de naturaleza inconsciente, que puede trabajarse exegéticamente.

Es claro que, en tanto disciplina hermenéutica, el psicoanálisis se sitúa más allá de los hechos manifiestos u objetivos, fisiológicos e incluso psicológicos, extrañándose si se pretende una validación de sus presupuestos y hallazgos con el metro empírico. No quiere ello decir que el psicoanálisis se oponga a ser tratado de esta forma en determinados aspectos de su teoría y de su quehacer, pero, como ha escrito el citado Ricoeur (19), no es lo mismo prestarse a una valoración empírica que hacer posible la contrastación empírica de una interpretación que se centra en la semántica del deseo: en este caso, las conjeturas psicoanalíticas han de considerarse bajo la condición de una probabilidad semántica del deseo, lo que no es igual que la probabilidad de un hecho observable por los órganos de los sentidos. 
Tal planteamiento ha llevado a algunos a mantener que, al fin y a la postre, el soporte epistemológico del psicoanálisis es semejante al de la fenomenología, de modo que ambos enfoques serían similares. Ricoeur $(19,26)$ llama la atención sobre este error, pues aunque psicoanálisis y fenomenología tengan algunos puntos comunes (como el acto filosófico de la reducción, las implicaciones de los aspectos dialécticos del lenguaje, la intersubjetividad que ambos comportan y los componentes históricos que a los dos interesan), la fenomenología es esencialmente una disciplina reflexiva, mientras que el psicoanálisis no lo es (si acaso autorreflexiva); además, el desplazamiento metodológico que el psicoanálisis efectúa difiere bastante de la estricta reducción fenomenológica, persiguiendo asir con la interpretación el significado inconsciente del acto conductual, a diferencia de la fenomenología que se detiene en la comprensión vivencial, sin ir nunca más allá de la esfera de lo consciente.

Psicoanálisis y fenomenología, en todo caso, son dos quehaceres hermenéuticos, pero con planteamientos, compromisos y metodologías bien distintos. La hermenéutica psicoanalítica busca la traducción de un texto manifiesto, que ha sido deformado, alterado, censurado y oscurecido, engañando al propio sujeto: el encuentro con el último sentido lo hace el psicoanalista contando con los determinantes inconscientes, que se consideran las principales claves del carácter y de la conducta. Por ello, el eje central de su método y de su técnica es la interpretación, superando la manifestación incompleta o distorsionada del texto consciente.

Habermas (27), en una línea argumental semejante, nos dice que el psicoanálisis se presenta aparentemente como una hermenéutica que se atiene al modelo filológico que utiliza el fenomenólogo, pero si se mira atentamente el trabajo interpretativo del analista se distingue bastante del que el fenomenólogo lleva a cabo, no sólo por su particular objeto de interés, sino especialmente porque tiene muy en cuenta la dimensión inconsciente, tratando de ir más allá del elemento manifiesto asentado en la consciencia, que es donde se detiene el fenomenólogo. El psicoanálisis, sigue diciendo Habermas, se consolida como una tecnología particular porque Freud capta, en las acciones y expresiones aparentemente discordantes del neurótico, una intención, un sentido, un significado, cuyo origen se hunde en el inconsciente: la interpretación analítica se ocupa, así, de aquellos contenidos deformados, alterados y mutilados a través de los que el sujeto se engaña a sí mismo, con lo que la hermenéutica filológica de Dilthey se transforma en el psicoanálisis en una hermenéutica de lo profundo.

La hermenéutica psicoanalítica, por otra parte, no tiene como objetivo final la mera comprensión intelectual de contenidos simbólicos, como acontece en las tradicionales ciencias del espíritu, sino que su meta principal está en lograr que el sujeto alcance una autocomprensión saturada de afectos (insight): no basta, e incluso desde el punto de vista terapéutico es inútil, que el psicoanalista proponga un significado del acto o del contenido mental y que el analizado lo acepte intelectualmente, puesto 
que es imprescindible que éste, superando las barreras de las defensas/ resistencias, tenga ocasión de experimentar el jah! cognitivo y afectivo que indica que su yo ha alcanzado la total comunicación con contenidos antes inaccesibles (27).

Finalmente, el psicoanálisis necesita de un complejo proceso de translaboración, por medio del cual pueden superarse todas las resistencias y asumirse los contenidos reprimidos: el psicoanálisis no busca, pues, un mero análisis seguido de una síntesis, sino lo que Habermas (27) bautizó como autorreflexión y Lorenzer $(28,29)$ como tarea crítico-hermenéutica, que en lenguaje psicoanalítico sería hacer consciente lo inconsciente, pasar lo que está regido por el proceso primario al proceso secundario o cambiar el dominio del ello en dominio del yo, relacionando lo expresado con los oportunos elementos inconscientes (30), transformando lo ignoto en conocido y lo ingobernable en controlable. Semejante idea late en la fórmula lacaniana que sitúa como meta pasar al orden simbólico lo que está en el orden imaginario.

Cuestión aparte es que la hermenéutica aún está lejos de establecer un cuerpo de principios bien asentados y contrastados, lo que aboca en una afirmación que algunos se resisten a pronunciar: siendo el saber psicoanalítico científico, es todavía muy inmaduro y provisional. Pero, en todo caso, no es en el terreno empírico-experimental, ni en leyes deterministas que permitan una explicación causal donde está su lugar, ni tampoco en el hallazgo de un cuerpo de principios que justifiquen ciertas explicaciones funcionales o en reconstrucciones de la esfera de lo consciente que aboquen en una explicación histórico-comprensiva de los hechos, sino en una categoría distinta de saberes científicos: en lo histórico-hermenéutico, campo dotado de un cuerpo de principios generales y particulares que puedan ser un buen pilar para las explicaciones interpretativas a la luz de los determinantes inconscientes.

\section{ESTRUCTURA LÓGICA DE LA ACTIVIDAD PSICOANALÍTICA}

Es necesario plantear ahora la estructura lógica de la actividad psicoanalítica, que afecta tanto a la reconstrucción histórica como a la estricta interpretación, así como las posibilidades de la contrastación de sus hallazgos, todo lo cual supone enfrentarse a los fundamentos epistemológicos de estos instrumentos técnicos, claves en el psicoanálisis en cualquiera de sus facetas. En tal sentido, se implican al menos tres aspectos particulares, que suponen tres problemas epistemológicos distintos: el carácter explicativo de la reconstrucción y de la interpretación, el asunto referido al aspecto semántico de ambas y la vertiente instrumental (especialmente terapéutica) de los dos instrumentos.

Vamos a tratar de mostrar que la actividad del psicoanalista se sedimenta en una estrategia común a la que usan otros investigadores científicos, aunque poseyendo determinadas peculiaridades que impiden la obtención de un rango epistemológico indiscutible. 


\section{LA CARA EXPLICATIVA DE LA RECONSTRUCCIÓN Y DE LA INTERPRETACIÓN}

Como ha dicho Klimovsky (31), el asunto relacionado con la naturaleza explicativa de la interpretación psicoanalítica (y para nosotros también el relacionado con la reconstrucción histórica) es de orden gnoseológico, debiéndose relacionar con el tipo de conocimiento que ocasiona. Podemos afirmar al respecto que tales intervenciones suponen habitualmente conjeturas con una pretensión explicativa (histórica, económica, dinámica) o comprensiva (semántica) sobre determinados fenómenos conductuales humanos, lo que trae consigo importantes cuestiones epistemológicas. En tal sentido, lo primero a afirmar es que la reconstrucción histórica o la interpretación psicoanalíticas, cualquiera que sea su contenido, suponen sin duda un acto de conocimiento, una especie de afirmación que hace el psicoanalista respecto al contenido o significado de determinado material verbal, o en general conductual, observado en el analizado, pudiendo también adelantarse a la aparición de tal material, en función de vivencias contratransferenciales y de ciertos elementos teóricos que asume.

En todo caso, cuando el psicoanalista reconstruye o interpreta formula una proposición o sentencia declarativa, algo que puede ser acertado o equivocado. En algunos casos, además, tal proposición es de naturaleza hipotética, desconociéndose en principio la dosis de verdad o falsedad que posee, duda que afecta tanto al psicoanalista como al psicoanalizado. Solamente, y con todas las limitaciones que ello conlleva, tras captar uno y otro los efectos que sus intervenciones traen consigo en los nuevos datos que aparecen, la reconstrucción y la interpretación empiezan a perder su carácter conjetural, para permitir su posible confirmación o refutación.

El psicoanalista trata con dos tipos de contenidos, el manifiesto (que puede ser estimado de naturaleza empírica) y el latente (que es de naturaleza distinta, pues no puede ser observado por los sentidos). Es cierto que el psicoanalista mantiene que ambos contenidos están íntimamente relacionados, pero, en todo caso, es evidente que entre los dos hay un salto epistemológico, lo que se capta cuando se intenta sus respectivas descripciones. De tal asunto deriva, evidentemente, uno de las cuestiones epistemológicas más nucleares del psicoanálisis: ¿cómo es posible fundamentar el conocimiento del inconsciente (material de tipo latente) y de qué manera debe ser ordenado y sistematizado este saber, habida cuenta que se trata de algo inferido o supuesto, y no de una realidad directamente observable?

Debe tenerse en cuenta, en este asunto, que cuando se reconstruye o se interpreta a partir de lo manifiesto, no se está describiendo ni correlacionando, ni se está colocando un hecho descriptivo en el contexto de otro hecho descriptivo, sino que se está transcendiendo del dato empírico al dato teórico, de lo objetivo a lo subjetivo, incluso de lo físico a lo psíquico. ¿Cómo es ello posible? Para aclarar esta cuestión es útil recurrir al esclarecimiento de las estrategias que sigue el psicoanalista en su quehacer: la inductiva y la deductiva. 


\section{LA ESTRATEGIA INDUCTIVA}

En la investigación científica existen varios procedimientos para tener acceso a lo que no es directamente cognoscible, desde la utilización de soportes tecnológicos sofisticados hasta el uso de métodos exegéticos. Pero, en todo caso, hay que contar con una teoría y con las oportunas reglas de correspondencia (aportadas desde luego por el propio sistema teórico) entre las construcciones derivadas de la teoría y los hechos sensibles, para así poder ir más allá de lo físicamente observable, es decir, del material empírico o manifiesto: tal acto supone la utilización de una estrategia de naturaleza inductiva.

Pues bien, la reconstrucción histórica o la interpretación psicoanalíticas pueden apoyarse, y de hecho así acontece, en los hechos conductuales manifiestos que se observan en el analizado (palabras, gestos, etc.), induciendo cuál fue la realidad vivida o fantaseada en el pasado y su significado a la luz de la dinámica inconsciente, contando con la oportuna teoría y con las adecuadas reglas de correspondencia. En ocasiones, lo observado (que podemos llamar 0 ) se vincula con lo inconsciente conjeturado (que denominaremos Ic) mediante la conocida regla si 0, entonces Ic. Un empirista rígido afirmaría que, en tal caso, lo único que deberíamos afirmar es que conocemos 0 , debiéndonos limitar a describirlo, lo que conlleva que todo acto de conocimiento no supone ningún sustrato teórico. Para otros, sin embargo, todo genuino conocimiento científico implica algún concepto teórico (aun contando con que el investigador lo desconozca o lo niegue), pues si lo teórico no subyace es imposible captar algo significativo: así, cuando se observa un material celular a través de un microscopio, no se verá nada con sentido, salvo que se tenga en la cabeza alguna construcción teórica sobre la citología.

Evidentemente, igual que cualquier otro científico, el psicoanalista efectúa sus inducciones en función de su marco teórico, traduciendo que tras 0 está Ic, con todas las limitaciones que derivan de la amplitud y riqueza de la conducta y de la parcialidad y provisionalidad de sus teorías. En tal lectura, cuando se trata del compromiso histórico, la regla de correspondencia sería si tenemos un elemento ' 0 ', tiene que estar también un elemento 'Ic' del pasado y si el compromiso es hermenéutico, la regla de correspondencia que se maneja sería si tenemos un signo ' 0 ', constituido por elementos observables, tal signo está en el sentido 'Ic', no observable. En cualquier caso, se entiende que 0 es condición suficiente para Ic y que Ic es condición necesaria para 0 , relación legal que enseña la teoría, que tiene todas las posibilidades de error que se quieran, pero que es perfectamente asumible desde la epistemología.

\section{LA ESTRATEGIA DEDUCTIVA}

La legalidad más profunda y consistente es, sin embargo, inversa a la discutida, asentando en una estrategia deductiva, también usual en la investigación y práctica psicoanalíticas, valiéndose de la argumentación que mantiene que un contenido 
latente 0 inconsciente de naturaleza hipotética (HIc) es la condición suficiente y que el contenido manifiesto ( 0 ) es la condición necesaria: esto es, si existe un determinado material inconsciente (HIc) se dará, probablemente, una concreta conducta manifiesta (0). En este caso se está llevando a cabo una estrategia deductiva que se atiene al modelo de cobertura legal de Hempel, de categoría epistemológica superior a la inductiva.

En efecto, tanto en la reconstrucción-explicación histórica como en la interpretación-explicación hermenéutica se pone sobre el tapete una hipótesis y se deducen una serie de consecuencias, con arreglo a un principio general o unos principios concretos, siendo posible verificar si aquella conjetura se cumple o no, a partir de la observación de los hechos que aparecen en la conducta manifiesta, o, en el caso terapéutico, teniendo en cuenta la finalidad instrumental del psicoanálisis, esto es, los efectos positivos que se establecen.

No puede negarse que la teoría psicoanalítica es una rica fuente de hipótesis y deducciones, sometibles a una eventual contrastación, habitualmente teniendo en cuenta el contexto clínico, aunque también es posible la contrastación observacional sin más, como se efectúa en el terreno evolutivo, sociológico, etc. En todo caso, en el encuentro psicoanalítico de naturaleza terapéutica es donde más adecuadamente se puede llevar a cabo la verificación-refutación de las conjeturas analíticas, sucediendo las cosas aproximadamente así: cuando un psicoanalista ha recogido, por medio de una peculiar observación participante, determinada cantidad de material manifiesto, a través de un procedimiento racional y también intuitivo se plantea las eventuales hipótesis explicativas, ya históricas, ya hermenéuticas, tratando después de advertir cuáles de ellas se ajustan mejor a los elementos en juego. Esto plantea, sin duda, nuevos problemas epistemológicos, pues los que crearon la lógica ya dijeron que sus leyes sólo garantizan que si se parte de verdades se llegue a verdades, pero advirtieron también que, aun razonando correctamente, de lo falso puede deducirse lo verdadero. 0 dicho de otra forma: la lógica no garantiza nada acerca de lo que pasa si se parte de hipótesis equivocadas, pues no es imposible que desde ellas se deriven consecuencias verdaderas, y éstas no garantizan de ningún modo la validez de las conjeturas manejadas.

En el trabajo psicoanalítico hay, además, otros inconvenientes, que antes se insinuaron, a la hora de enjuiciar su valor científico: dado que la reconstrucción histórica y la interpretación dinámica suponen un decir, un desvelar al sujeto lo que se presume, esto puede condicionar los hechos siguientes, que son los que confirmarán o refutarán aquéllas. Puede suceder, incluso, que siendo correcta la reconstrucción o la interpretación, el sujeto las rechace por la actuación de resistencias inconscientes u otras circunstancias; pero también puede acontecer que, siendo erróneas, las confirme, por razones transferenciales o de otro orden. Esto hace que las reconstrucciones e interpretaciones psicoanalíticas en ocasiones se tornen autopredictivas, y por ello de alguna manera suicidas frente a su contrastación científica. 


\section{LA CARA SEMÁNTICA DE LA RECONSTRUCCIÓN Y DE LA INTERPRETACIÓN}

El carácter semántico o comprensivo del psicoanálisis tiene que ver con la función simbólica o de signo que está contenida en los más variados actos conductuales manifiestos, ya se trate de una asociación verbal libre, un lapsus, un acting-out, un síntoma, un rasgo caracterial, un fenómeno transferencial, etc. En tal sentido, reconstruir e interpretar supone un ejercicio estrictamente hermenéutico, un acto mediante el cual se trata de dar sentido o significado (precisamente el determinado por el inconsciente, contando con el ambiente y la cultura en que se ha desenvuelto el sujeto) a un significante concreto, justamente el formado por el hecho reconstruido o expresado en el campo de las relaciones transferenciales o en menor medida en la vida diaria del sujeto. Naturalmente, todo esto implica que lo manifiesto no sólo posea una relación legal con lo latente, sino también una relación de sentido.

En este plano, el más genuino del psicoanálisis, nos enfrentamos con lo que podríamos llamar la semiótica psicoanalítica, que es un campo en desarrollo, no habiendo logrado aún una suficiente madurez, como lo expresa el hecho que viene dado por las distintas interpretaciones que pueden darse a un determinado significante en función de los posicionamientos teóricos asumidos. Esto se complica, además, por el hecho de que siempre se da una multideterminación del signo, interviniendo en ello no sólo las peculiaridades individuales, sino también las culturales e históricas, personales y de toda la humanidad, como las aportaciones junguianas han revelado, aunque éstas insisten en ciertos elementos comunes y universales. En cualquier caso, todo ello conduce al carácter altamente probabilístico de la semántica del hecho interpretado: de aquí la insistencia psicoanalítica en evaluar la interpretación en el contexto metodológico dado por su sistema, que procura la observación reiterada, la acumulación de datos, el profundo conocimiento biográfico y la liquidación de las resistencias que impiden al analizado el acceso al significado inconsciente del elemento en cuestión.

Naturalmente, al efectuar la tarea de dar sentido a lo psicológico, también se sirve el psicoanalista de una estrategia inductiva o deductiva. Al respecto, ha de admitirse que, tanto en una como en otra estrategia, o en la búsqueda de explicaciones históricas o explicaciones exegéticas, en el psicoanálisis se da una cobertura epistemológica laxa, y, consiguientemente, contestable desde posicionamientos científicos más maduros. Pero esto no quiere decir, de ninguna forma, que el trabajo psicoanalítico reconstructivo e interpretativo sea un juego azaroso ajeno a los presupuestos de una actividad investigadora, que se maneja con fines manipuladores o interesados. Hacer equivalentes la magia y el psicoanálisis es una actitud emprejuiciada donde uno está tentado de traer a colación, por mucho que quiera evitarlo, el argumento ad hominem. Más razonable sería, quizás, mantener que el psicoanálisis es, en ciertos aspectos, una contraciencia, que pone en crisis los supuestos tradicionales de las ciencias positivas. 


\section{ASPECTO INSTRUMENTAL DE LA RECONSTRUCCIÓN Y DE LA INTERPRETACIÓN}

La reconstrucción y la interpretación psicoanalíticas, por último, tienen compromisos instrumentales, de naturaleza más terapéutica que investigadora o científica. Ciertamente, el psicoanalista, en su quehacer cotidiano, no maneja las reconstrucciones e interpretaciones buscando el hallazgo de legalidades científicas, sino especialmente intentando originar una serie de efectos clínicos deseables, a partir de los cambios metapsicológicos que pone en marcha en los conflictos inconscientes del analizado. La teoría y la práctica psicoanalíticas mantienen que el trabajo psicoanalítico, y en particular la interpretación, ocasiona que los contenidos inconscientes, que mantienen activos los conflictos, puedan pasar a la esfera de lo consciente, al proceso secundario $o$ al dominio del yo, a través de la liquidación de las defensas/resistencias y de los oportunos insights, así como de las acompañantes experiencias emocionales correctoras.

Pues bien, así como el principal problema epistemológico en el campo de la gnoseología era encontrar la verdad, lo decisivo en el terreno instrumental es lograr un cambio caracterial y conductual rápido, estable y adecuado, es decir, una intervención terapéutica efectiva, eficaz y eficiente, criterio de orden tecnológico y no estrictamente científico. Por consiguiente, el tema de la instrumentalidad se liga al de la técnica. Y en este sentido, el psicoanálisis ha establecido con bastante precisión las condiciones y los contenidos del proceso terapéutico, los instrumentos de trabajo, los objetivos inmediatos y lejanos y las anomalías y las etapas del curso de la terapia, así como los caminos necesarios para adquirir la técnica. Así mismo, se ha buscado y se busca una contrastación objetiva de los cambios terapéuticos (resultados, procesos), lo que ha alcanzado recientemente un nivel muy aceptable. Pero, en todo caso, no puede olvidarse que el psicoanálisis, como intervención terapéutica, supone un encuentro humano, con todos los ingredientes de espontaneidad, de respeto, de genuino amor e incluso de arte que tal hecho debe traer aparejado. Y todo esto, desde luego, escapa a los parámetros de la ciencia.

\section{BIBLIOGRAFÍA}

1. Sánchez-Barranco, A., Naturaleza histórico-hermenéutica del saber psicoanalítico, Revista de Historia de la Psicología, 3-4, 1991, 351-355.

2. Sánchez-Barranco, A., La condición científica del psicoanálisis, Apuntes de Psicología, 36, 1992, 57-76.

3. Sánchez-Barranco, A., Naturaleza de la investigación psicoanalítica: reflexiones epistemológicas, III Jornadas de Psicoanálisis en la Universidad, Gerona, noviembre 1992.

4. Bridgman, P.W., Operational Analysis, Philosophy of Science, 5, 1938, 114-131. 
5. Bridgman, P.W., Some General Principles of Operational Analysis, Psychological Review, 52, 1945, 246-249.

6. Madison, P., Freud's Concept of Repression and Defense. Its Theoretical and Observational Language, Minneapolis, Minnesota University Press, 1961.

7. Sullivan, H.S., The Interpersonal Theory of Psychiatry, New York, Norton, 1953.

8. Sullivan, H.S., The Fusion of Psychiatry and Social Science, New York, Norton, 1964.

9. Fine, R., Historia del psicoanálisis, 2 tomos, 1979, Buenos Aires, Paidós, 1982.

10. Chrzanowski, G., International Approach to Psychoanalysis, New York, Wiley, 1977.

11. Rapaport, D., El modelo conceptual del psicoanálisis. En El modelo psicoanalítico, la teoría del pensamiento y las técnicas proyectivas, 1951, Buenos Aires, Hormé, 1978, 9-44.

12. Rapaport, D., La estructura de la teoría psicoanalítica, 1960, Buenos Aires, Paidós, 1971.

13. Peterfreund, E., The Need for a New Theoretical Frame of Reference for Psychoanalysis, Psychoanalytic Quarterly, 44, 1975, 534-549.

14. Peterfreund, E. y Schwartz, J.T., Información, sistemas y psicoanálisis, 1971, México, Siglo XXI, 1976.

15. Gear, M. y Liendo, E., Acción psicoanalítica, Caracas, Monte Ávila, 1976.

16. Gear, M. y Liendo, E., Informática psicoanalítica, Buenos Aires, Nueva Visión, 1977.

17. Klein, G.S., Psychoanalytic Theory, New York, International University Press, 1976.

18. Schafer, R.S., A New Language for Psychoanalysis, New Haven, Yale University Press, 1976.

19. Ricoeur, P., Freud: una interpretación de la cultura, 1965, México, Siglo XXI, 1975.

20. Sánchez-Barranco, A. La condición científica del psicoanálisis. Consideraciones epistemológicas, Sevilla, Universidad de Sevilla, 1986.

21. Sánchez-Barranco, A., El psicoanálisis, una ciencia hermenéutica. En La psicología hoy. De la teoría a la intervención, Sevilla, Universidad Nacional de Educación a Distancia, monografía 0, 1986, 9-27.

22. Sánchez-Barranco, A., Historia y fundamentos (conceptuales y epistemológicos) de la técnica psicoanalítica, Sevilla, Departamento de Psicología Evolutiva y de la Educación, Básica y Metodología (Universidad de Sevilla), 1988.

23. Sánchez-Barranco, A., Técnica psicoanalítica. Desarrollo histórico, conceptos fundamentales y fundamentos epistemológicos, Sevilla, Arquetipo, 1993.

24. Gibson, Q., La lógica de la investigación social, 1959, Madrid, Tecnos, 1982. 
25. Suárez, A., Nota preliminar. En P. Ricoeur, Freud: una interpretación de la cultura, México, Siglo XXI, IX-XI, 1975.

26. Ricoeur, P., Hermenéutica y psicoanálisis, 1969, Buenos Aires, La Aurora, 1979.

27. Habermas, J., Conocimiento e interés, 1968, Madrid, Tecnos, 1982.

28. Lorenzer, A., El lenguaje destruido y la reconstrucción psicoanalítica, 1970, Buenos Aires, Amorrortu, 1977.

29. Lorenzer, A., Bases para una teoría de la socialización, 1972, Buenos Aires, Amorrortu, 1976.

30. Fornari, F., Teoría semiótica de la interpretación psicoanalítica. En L. Grinberg (comp.), Prácticas psicoanalíticas comparadas en las neurosis, Buenos Aires, Amorrortu, 1977, 46-47.

31. Klimovsky, G., Aspectos epistemológicos de la interpretación psicoanalítica. En E.R. Etchegoyen, Los fundamentos de la técnica psicoanalítica, Buenos Aires, Amorrortu, 1986, 433-456.

Antonio Sánchez-Barranco Ruiz

Psiquiatra

Departamento de Psicología Experimental

Universidad de Sevilla. Sevilla. España

Pablo Sánchez-Barranco Vallejo

Psiquiatra

Department of Psychiatry

New York University School of Medicine

Bellevue Hospital Center. New York, NY, USA.

Fernando Sánchez-Barranco Vallejo

Médico

Sevilla. España

Correspondencia:

Antonio Sánchez-Barranco Ruiz

Pza. Dr. González Gramage, 2-1º B

41005 Sevilla 